\title{
Survivin and gliomas: A literature review
}

\author{
ROSILIN KOTAKKATHU VARUGHESE ${ }^{1}$ and SVERRE HELGE TORP ${ }^{1,2}$ \\ ${ }^{1}$ Department of Laboratory Medicine, Children's and Women's Health, \\ Faculty of Medicine, Norwegian University of Science and Technology (NTNU), 7030 Trondheim; \\ ${ }^{2}$ Department of Pathology and Medical Genetics, St. Olavs Hospital, 7006 Trondheim, Norway
}

Received July 28, 2015; Accepted May 10, 2016

DOI:10.3892/ol.2016.4867

\begin{abstract}
Gliomas are the most common primary brain tumor, the diagnosis of which is challenging. In this respect, the use of immunohistochemical proliferation markers may aid diagnosis; survivin, also known as Baculoviral IAP Repeat Containing 5, is one such marker. Survivin is a unique member of the inhibitors of apoptosis protein gene family, and is known for its dual function as an apoptosis inhibitor and mitosis regulator. Furthermore, survivin has been demonstrated to be overexpressed in a number of malignancies. The purpose of the present literature review was to gain an overview of studies published on the diagnostic and/or prognostic use of survivin in gliomas. Using PubMed, 19 studies matching the inclusion criteria were ultimately included in the present review. The majority of the studies identified revealed that survivin was significantly associated with other proliferation markers, histological malignancy grade, and inversely associated with prognosis. However, there were a number of inconsistencies between studies, which suggests a requirement for standardization of immunohistochemical procedures.
\end{abstract}

\section{Introduction}

There is a multitude of histological types of brain tumors, which are classified according to the World Health Organization (WHO) (1). Gliomas are one such entity, and consist of astrocytomas, oligodendrogliomas, ependymomas and gangliogliomas. Overall, gliomas are the most common primary malignant intracerebral neoplasm with an incidence rate of 6.03 per 100,000 individuals every year (2). Classification and malignancy grading may be challenging, and there is constant investigation for improved methods to aid neuropathologists. In this respect, immunohistochemistry has received a lot of

Correspondence to: Dr Rosilin Kotakkathu Varughese, Department of Laboratory Medicine, Children's and Women's Health, Faculty of Medicine, Norwegian University of Science and Technology (NTNU), Erling Skjalgssons Gate 1, 7030 Trondheim, Norway

E-mail: rosilinv@gmail.com

Key words: survivin, BIRC5, immunohistochemistry, astrocytomas, ependymomas, oligodendrogliomas attention, and is a fundamental tool in the daily routine of the majority of pathology departments. Immunohistochemistry remains an important modality for glioma diagnosis, since it is a robust, convenient and affordable method, with which the majority of laboratories have considerable experience. Other relevant methods for diagnosing gliomas are molecular genetics, including fluorescence in situ hybridization and polymerase chain reaction. Indeed, all these techniques in combination are essential for classifying tumors in accordance with the newly published classification scheme of tumors of the central nervous system by the WHO (1).

Survivin, also known as Baculoviral IAP Repeat Containing 5, is a member of the inhibitors of apoptosis protein gene family. It is considered unique for its dual function as an apoptosis inhibitor and mitosis regulator $(3,4)$. Survivin is generally only expressed during tissue development, and although it is observed in certain normal tissues with high proliferative activity, it is scarce in the majority of adult tissues (3). By contrast, survivin is overexpressed in numerous malignancies, including lung, pancreatic, breast, ovarian and colon cancer $(3,5-8)$. Therefore, survivin has clinical potential; not only is it considered as an immunohistochemical diagnostic and prognostic marker, but it has also been identified as a potential target for therapy (3).

The aim of the present study is to provide a literature review on the use of survivin as an immunohistochemical marker in gliomas.

\section{Materials and methods}

Inclusion criteria. The present literature review was restricted to studies concerning human gliomas published in English during the last 10 years; therefore covering 2004-2014. Other inclusion criteria consisted of studies that focussed either on the prognostic and/or diagnostic value of survivin, and included a minimum of 15 patients.

Search terms. Studies were identified using the search engine PubMed (www.ncbi.nlm.nih.gov/pubmed). To identify relevant studies a meticulous set of search terms was constructed. The final search parameter included the terms 'survivin', 'gliomas', 'astrocytomas', 'oligodendrogliomas', 'ependymomas' and 'gangliogliomas'.

The full search code used is as follows and this yielded 202 studies: Survivin[All Fields] AND (("glioma"[MeSH 
Terms] OR "glioma”[All Fields] OR "gliomas"[All Fields]) OR ("astrocytoma"[MeSHTerms]OR "astrocytoma"[AllFields]OR "astrocytomas"[All Fields]) OR ("oligodendroglioma"[MeSH Terms] OR "oligodendroglioma"[All Fields] OR "oligodendrogliomas"[All Fields]) OR ("ependymoma"[MeSH Terms] OR "ependymoma"[AllFields] OR "ependymomas"[All Fields]) OR ("ganglioglioma"[MeSH Terms] OR "ganglioglioma"[All Fields] OR "gangliogliomas"[All Fields])) AND ((“2004/01/01”[PDAT] : “2014/12/31”[PDAT]) AND English[lang]).

Exclusion criteria. Based on title and abstracts 183 studies were excluded, leaving 19 studies for further evaluation. Exclusion criteria were animal studies, meta-analyses, tumors that were not gliomas, such as medulloblastomas, and studies where the primary focus was the detection of survivin by means other than immunohistochemistry.

\section{Results}

Survivin expression. Table I presents a summary of the 19 studies included in the present review (9-27). The majority of studies observed nucleic and cytoplasmic immunoreactivity for survivin expression in gliomas; however, there were differences in which expression was used for analysis. Whereas certain studies included, and even separately considered, the two forms of expression (9-14), other studies focused solely on nuclear staining (15-21). Furthermore, certain studies did not explicitly state which form was assessed (22-27).

In general, the studies used one of three methods for quantifying survivin in gliomas: Labeling index (LI), staining intensity (SI) or immunoreactivity score (IRS). LI, defined as the percentage of immunoreactive tumor cells out of the total number of cells, was used by 10/19 (52.6\%) studies (14-16,18-24). The majority of studies that used SI applied a four-tiered system as follows: 0 , negative staining; 1 , weak staining; 2 , moderate staining; and 3, strong staining (10-14,17,25). Okada et al (26) and Yeung et al (27) used variations of this system, as detailed in Table I. Survivin IRS was determined by the multiplication of the percentage of survivin-positive cells $(0,<1 \% ; 1,1-25 \%$; $2,26-50 \% ; 3,51-75 \% ; 4,>75 \%)$ and the values for survivin SI $(13,17,25)$. Saito et al (9) focused specifically on the localization of survivin as being cytoplasmic and/or nuclear. In addition, that study recorded the staining as positive or negative, defined as $>5$ and $\leq 5 \%$, respectively.

Survivin and Ki67/MiB-1. In total, 10 studies (52.6\%) investigated whether there was an association between Ki67/MiB-1 expression and survivin in gliomas. The results varied as certain studies identified a significant association $(14,15,18-22)$, while others did not $(14,24,27)$. Saito et al (9) investigated whether the subcellular localization of survivin was associated with $\mathrm{Ki} 67 / \mathrm{MiB}-1$ expression, and demonstrated that it was not. Liu et al (14) reported a significant and a non-significant association with Ki67/MiB-1 for nuclear-survivin and cytoplasmic-survivin, respectively.

Survivin and tumor grades. The expression of survivin across histological malignancy grades was evaluated by $12 / 19$ (63.2\%) studies. A total of 8 out of the 12 studies (66.7\%) revealed that there was a greater survivin immunoreactivity with increasing tumor grade, and reported statistically significant differences in survivin expression across histological grades of gliomas $(13,14,16,20-22,24,25)$. However, 4 studies (33.3\%) identified no significant association between survivin and tumor grade $(9,17,26,27)$.

Survivin and survival. Out of the 19 studies, 14 (73.7\%) investigated the prognostic value of survivin. In total, 6 studies $(42.9 \%)$ were unable to demonstrate that survivin was significant in survival analysis $(15,18,19,21,25,26)$. By contrast, $8 / 14$ studies $(57.1 \%)$ reported that a high expression of survivin was inversely associated with prognosis $(9,11,12,16,20,22-24)$.

\section{Discussion}

The purpose of the present literature review was to provide an overview of the diagnostic and prognostic findings of survivin as an immunohistochemical marker in gliomas. In the majority of studies reviewed by the present study, survivin was positively associated with other proliferation markers, including Ki67/MiB-1, and histological malignancy grade, and inversely associated with prognosis. However, the data was encumbered with several elements of uncertainty that illustrate the requirement for a standardization of immunohistochemical procedures.

Expression and quantification of survivin. Cellular immunoreactivity for survivin is observed in the cytoplasm and nuclei, and it is very likely these forms of expression are associated with the dual function of survivin as an apoptosis inhibitor and a mitosis regulator, respectively (9-11). It has also been suggested that the difference in the subcellular localization of survivin varies according to the antibody used (18). The different immunohistochemical antibodies and the working dilutions used by the respective studies are listed in Table I. Kogiku et al (22) and Uematsu et al (24) did not use a commercial antibody, but prepared their own.

Although the majority of studies mentioned the localization pattern of the antibody, certain studies do not state this explicitly. Furthermore, as shown in Table I, there were differences as to which form of expression was registered (cytoplasmic or nuclear), how the expression of the marker was quantified and how this was considered statistically. Three primary quantification methods were used: LI, SI and IRS. Additionally, Saito et al (9) simply registered the subcellular localization of survivin as cytoplasmic, nucleic or both.

Notably, even between studies using LI there were certain discrepancies, such as the minimum number of cells that were evaluated ranged between 500 and 1,000. In addition, there are differences in the percentage of immunoreactive cells defined by the studies as 'high' or 'low' expression of survivin. As an example, one study used $</ \geq 3 \%$ as a low/high expression, while another used a cut-off of $\leq>>80 \%$; these studies were conducted on astrocytomas grades II-IV $(16,24)$. In numerous studies, the chosen cut-off appeared to be an arbitrary value.

The differences alluded to here, regarding expression and quantification, demonstrate the requirement of a standardization of procedures. The lack of specific guidelines allows for a variation of methods, which consequently renders it 


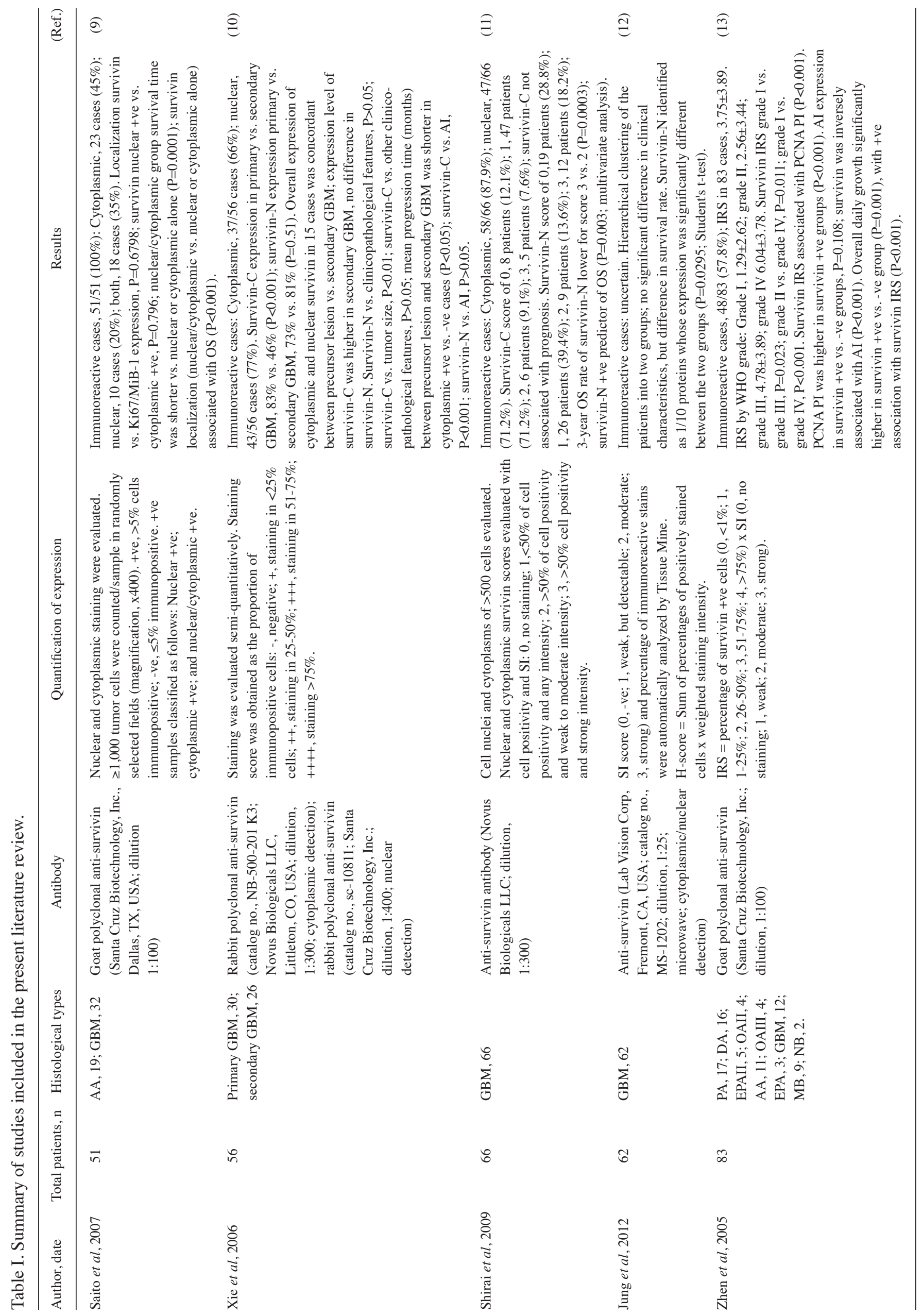




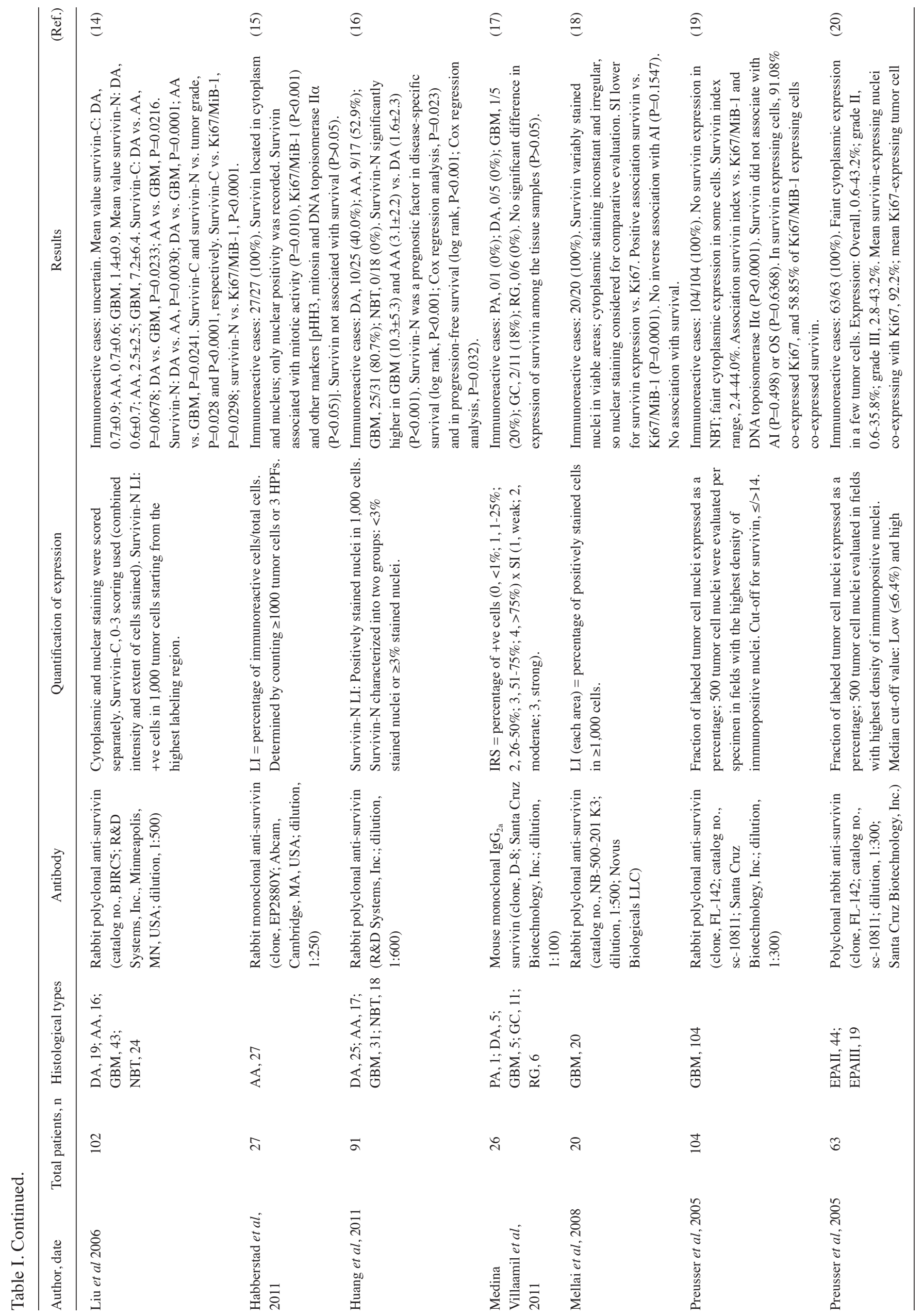




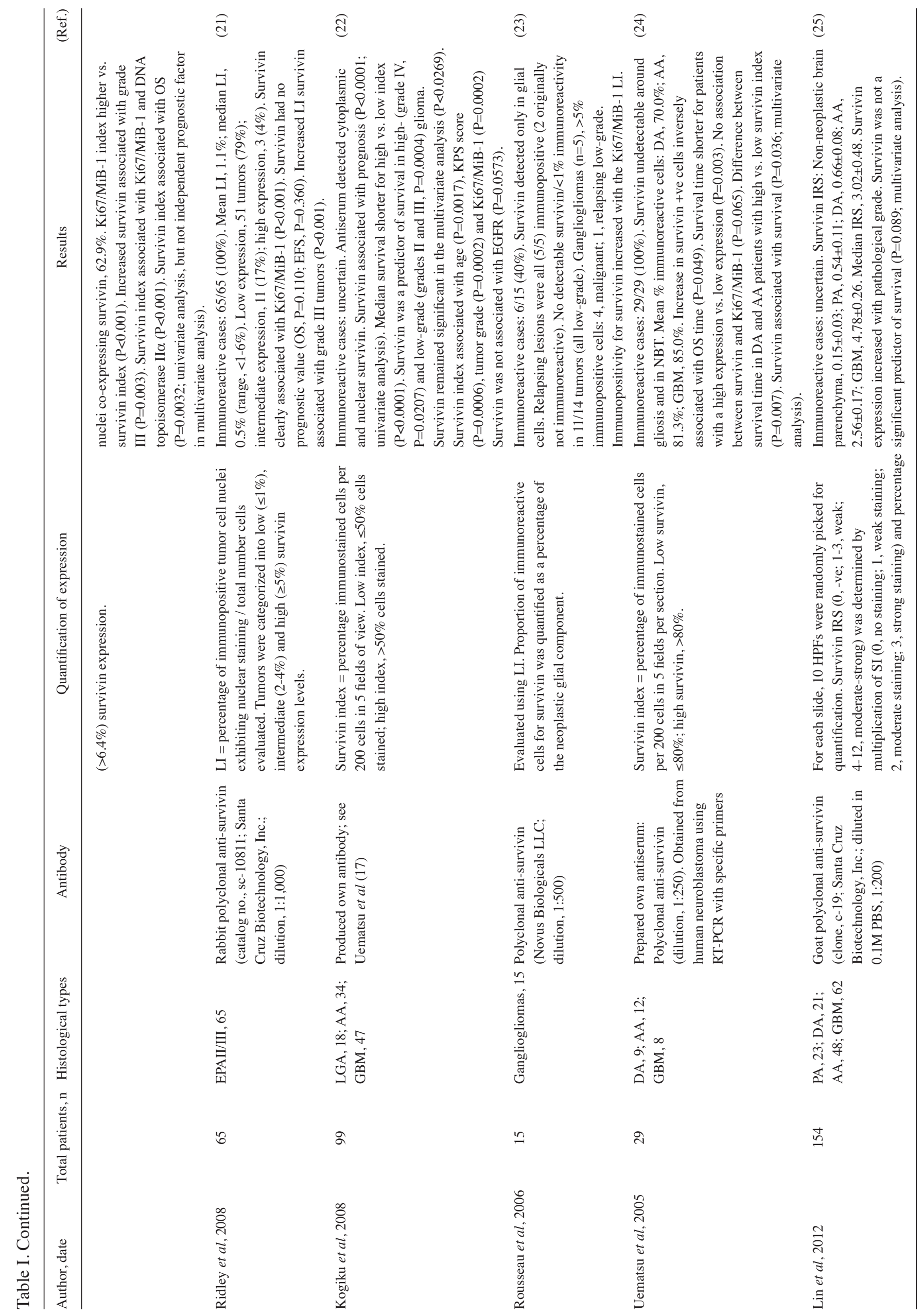




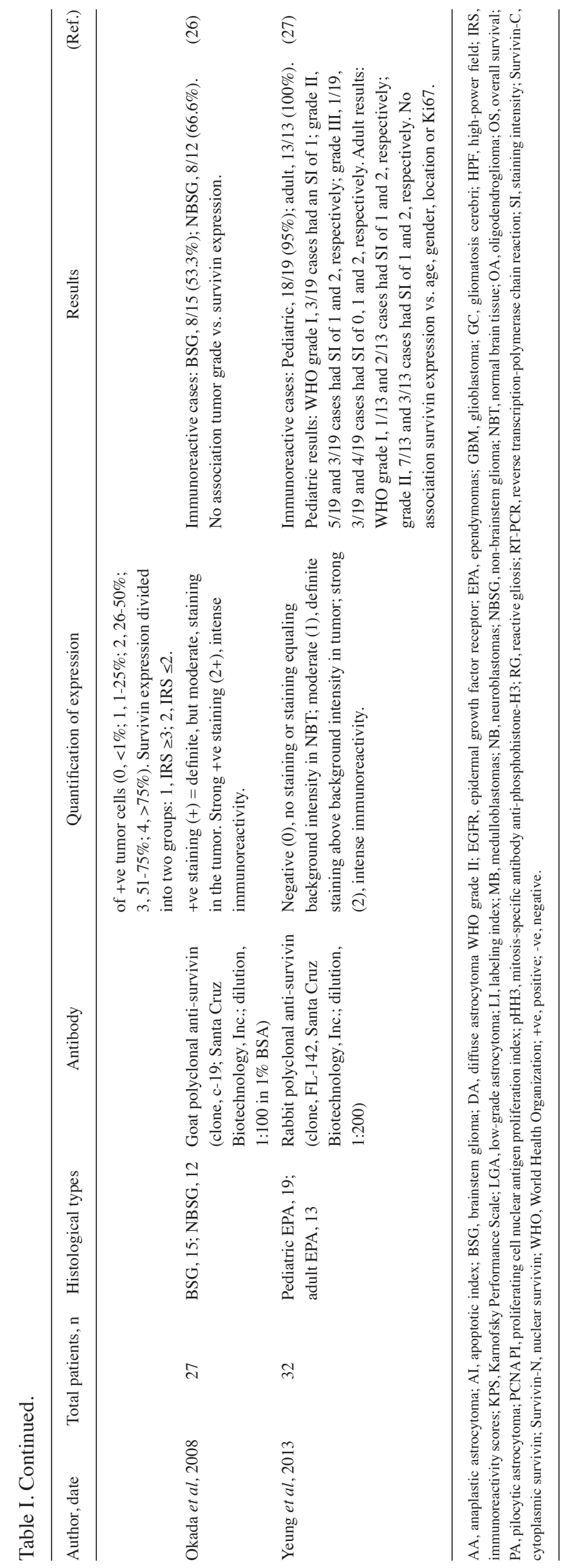


challenging to compare different studies and interpret the results in a practical and translatable manner. That said, this is one of the well-known shortcomings of immunohistochemistry; the same antibody used by different laboratories may often lead to a variety of results, making it challenging to set any definite parameters. This can be paralleled to studies using with Ki67/MiB-1 (28,29).

Survivin and $\mathrm{Ki} 67 / \mathrm{MiB}-1 . \mathrm{Ki} 67 / \mathrm{MiB}-1$ is a proliferation marker frequently used by pathologists to determine a tumor's proliferative activity. It is used in the diagnosis of various human malignancies, including breast cancer, neuroendocrine tumors and lymphomas. However, it is not officially recognized in the World Health Organization classification scheme of gliomas, since there is a considerable overlap in the proliferative indices of the marker between malignancy grades $(28,30)$.

The studies reviewed by the present study report positive $(14,15,18-22)$ and negative $(9,24,27)$ findings regarding an association between $\mathrm{Ki67} / \mathrm{MiB}-1$ and survivin. The majority of studies (70\%) suggest that there is a positive association $(14,15,18-22)$. A few studies report co-expression of the two markers, and reveal that Ki67/MiB-1 stained a significantly larger fraction of tumor cell nuclei compared with survivin (14,18-20). This is understandable, since survivin is only expressed in the $\mathrm{G} 2 / \mathrm{M}$ phase, whereas $\mathrm{Ki67} / \mathrm{MiB}-1$ is expressed in all phases of the cell cycle (20).

Uematsu et al (24) demonstrated that although the Ki67/MiB-1 index was significantly different between low-grade astrocytomas and glioblastomas, there was no difference in the LI between anaplastic astrocytomas and low-grade astrocytomas. By contrast, the survivin index was significantly different between anaplastic astrocytomas and low-grade astrocytomas, but not between glioblastomas and anaplastic astrocytomas. Based on these results the authors concluded that perhaps survivin may be a more sensitive marker compared with Ki67/MiB-1 in low-grade gliomas (24). However, Liu et al (14) reported that $\mathrm{Ki67/MiB-1} \mathrm{LI} \mathrm{and} \mathrm{nuclear-survivin} \mathrm{LI} \mathrm{are}$ significantly different between all grades of astrocytomas, and the differences observed with Ki67/MiB-1 are more significant compared with survivin. It is a point worth considering that Liu et al had 102 patients, while Uematsu et al had 29.

Survivin and tumor grades. Histological malignancy grade was considered in 12 studies, of which 8 reported that expression of survivin was associated with tumor grade $(13,14,16,20-22,24)$, and 4 identified no significant association $(9,17,26,27)$. Notably, all studies that report a significant association between survivin and grade use LI, except for Lin et al (25), who used IRS, and Liu et al (14), who used LI and SI. By contrast, none of the studies that reported negative findings used LI, but rather used variations of SI, IRS or simply a registration of the subcellular localization of immunoreactivity. Therefore, the findings indicate that survivin LI may indeed have some diagnostic potential. Future studies should take this into account as it demonstrates that objective measures of expression, including LI, are perhaps superior in a diagnostic setting. An important point is that, as with Ki67/MiB-1, survivin LI alone cannot and should not determine histological malignancy grade; the marker should always be used in combination with clinical information, which may aid pathologists in borderline cases (29).
Survivin and survival. The results regarding survivin and survival are ambiguous, and the previously mentioned heterogeneity of methods render it challenging to draw any clear conclusions. There is a slight majority of studies that report that a higher expression of survivin was significantly associated with poorer survival; however, these studies have various cut-off values for high and low expression of survivin $(9,11,12,16,20,22-24)$. Therefore, it is not entirely straightforward to interpret the results at face value.

Concluding remarks. Survivin is an immunohistochemical marker with potential in tumor diagnostics and prognostics, as well as a prospective therapeutic target (3). However, the challenges encountered using survivin in gliomas are largely the same as those met when utilizing other immunohistochemical markers, including Ki67/MiB-1 (28,29). For this reason, it would be beneficial to standardize immunohistochemical analysis and counting procedures, and establish standardized cut-off values. Ultimately, the end goal is to identify markers that may be used in combination with clinical information to optimize therapy for each individual patient.

\section{Acknowledgements}

The authors would like to thank Mr. Magnus B. Arnli [Children's and Women's Health, Faculty of Medicine, Norwegian University of Science and Technology (NTNU), Trondheim, Norway] and Dr Jobin K. Varughese (Haukeland University Hospital, Bergen, Norway) for critical revision and feedback on the manuscript.

\section{References}

1. Louis, DN, Ohgaki H, Wiestler OD, Cavenee WK, Ellison DA, Figarella-Branger D, Perry A, Reifenberger G and von Deimling A (eds): WHO Classification of Tumours of the Central Nervous System, Revised. 4th edition. Vol. 1. IARC, Lyon, 2016.

2. Dolecek TA, Propp JM, Stroup NE and Kruchko C: CBTRUS statistical report: Primary brain and central nervous system tumors diagnosed in the United States in 2005-2009. Neuro Oncol 14 (Suppl 5): v1-v49, 2012

3. Andersen MH, Svane IM, Becker JC and Straten PT: The universal character of the tumor-associated antigen survivin. Clin Cancer Res 13: 5991-5994, 2007.

4. Lens SM, Vader G and Medema RH: The case for Survivin as mitotic regulator. Curr Opin Cell Biol 18: 616-622, 2006.

5. Aune G, Stunes AK, Tingulstad S, Salvesen O, Syversen U and Torp SH: The proliferation markers Ki-67/MIB-1, phosphohistone $\mathrm{H} 3$, and survivin may contribute in the identification of aggressive ovarian carcinomas. Int J Clin Exp Pathol 4: 444-453, 2011.

6. Chen WB, Cheng XB, Ding W, Wang YJ, Chen D, Wang JH and Fei RS: Centromere protein $\mathrm{F}$ and survivin are associated with high risk and a poor prognosis in colorectal gastrointestinal stromal tumours. J Clin Pathol 64: 751-755, 2011.

7. Ekeblad S, Lejonklou MH, Stålberg P and Skogseid B: Prognostic relevance of survivin in pancreatic endocrine tumors. World J Surg 36: 1411-1418, 2012.

8. Zhang LQ, Wang J, Jiang F, Xu L, Liu FY and Yin R: Prognostic value of survivin in patients with non-small cell lung carcinoma: A systematic review with meta-analysis. PLoS One 7: e34100, 2012.

9. Saito T, Arifin MT, Hama S, Kajiwara Y, Sugiyama K, Yamasaki F, Hidaka T, Arita K and Kurisu K: Survivin subcellular localization in high-grade astrocytomas: Simultaneous expression in both nucleus and cytoplasm is negative prognostic marker. J Neurooncol 82: 193-198, 2007. 
10. Xie D, Zeng YX, Wang HJ, Wen JM, Tao Y, Sham JS and Guan XY: Expression of cytoplasmic and nuclear Survivin in primary and secondary human glioblastoma. Br J Cancer 94: 108-114, 2006

11. Shirai K, Suzuki Y, Oka K, Noda SE, Katoh H, Suzuki Y, Itoh J, Itoh $\mathrm{H}$, Ishiuchi $\mathrm{S}$, Sakurai $\mathrm{H}$, et al: Nuclear survivin expression predicts poorer prognosis in glioblastoma. J Neurooncol 91: 353-358, 2009

12. Jung Y, Joo KM, Seong DH, Choi YL, Kong DS, Kim Y, Kim MH, Jin J, Suh YL, Seol HJ, et al: Identification of prognostic biomarkers for glioblastomas using protein expression profiling. Int J Oncol 40: 1122-1132, 2012.

13. Zhen HN, Zhang X, Hu PZ, Yang TT, Fei Z, Zhang JN, Fu LA, He XS, Ma FC and Wang XL: Survivin expression and its relation with proliferation, apoptosis, and angiogenesis in brain gliomas. Cancer 104: 2775-2783, 2005.

14. Liu X, Chen N, Wang X, He Y, Chen X, Huang Y, Yin W and Zhou Q: Apoptosis and proliferation markers in diffusely infiltrating astrocytomas: Profiling of 17 molecules. J Neuropathol Exp Neurol 65: 905-913, 2006.

15. Habberstad AH, Gulati S and Torp SH: Evaluation of the proliferation markers Ki-67/MIB-1, mitosin, survivin, $\mathrm{pHH} 3$, and DNA topoisomerase II $\alpha$ in human anaplastic astrocytomas-an immunohistochemical study. Diagn Pathol 6: 43, 2011.

16. Huang Y, Chen X, Chen N, Nie L, Xu M and Zhou Q: Expression and prognostic significance of survivin splice variants in diffusely infiltrating astrocytoma. J Clin Pathol 64: 953-959, 2011.

17. Medina Villaamil V, Alvarez García A, Aparicio Gallego G, Díaz Prado S, Rivas López LA, Santamarina Caínzos I, Valladares Ayerbes M and Antón Aparicio LM: Tissue array analysis for the differentiation of gliosis from gliomas. Mol Med Rep 4: 451-457, 2011.

18. Mellai M, Caldera V, Patrucco A, Annovazzi L and Schiffer D Survivin expression in glioblastomas correlates with proliferation, but not with apoptosis. Anticancer Res 28: 109-118, 2008.

19. Preusser M, Gelpi E, Matej R, Marosi C, Dieckmann K, Rössler K, Budka H and Hainfellner JA: No prognostic impact of survivin expression in glioblastoma. Acta Neuropathol 109: $534-538,2005$

20. Preusser M, Wolfsberger S, Czech T, Slave I, Budka H and Hainfellner JA: Survivin expression in intracranial ependymomas and its correlation with tumor cell proliferation and patient outcome. Am J Clin Pathol 124: 543-549, 2005.
21. Ridley L, Rahman R, Brundler MA, Ellison D, Lowe J, Robson K, Prebble E, Luckett I, Gilbertson RJ, Parkes S, et al: Multifactorial analysis of predictors of outcome in pediatric intracranial ependymoma. Neuro Oncol 10: 675-689, 2008.

22. Kogiku M, Ohsawa I, Matsumoto K, Sugisaki Y, Takahashi H, Teramoto A and Ohta S: Prognosis of glioma patients by combined immunostaining for survivin, Ki-67 and epidermal growth factor receptor. J Clin Neurosci 15: 1198-1203, 2008

23. Rousseau A, Kujas M, Bergemer-Fouquet AM, van Effenterre R and Hauw JJ: Survivin expression in ganglioglioma. J Neurooncol 77: 153-159, 2006.

24. Uematsu M, Ohsawa I, Aokage T, Nishimaki K, Matsumoto K, Takahashi H, Asoh S, Teramoto A and Ohta S: Prognostic significance of the immunohistochemical index of survivin in glioma: A comparative study with the MIB-1 index. J Neurooncol 72: 231-238, 2005

25. Lin H, Wang Y, Zhang X, Liu B, Zhang W and Cheng J: Prognostic significance of kappaB-Ras1 expression in gliomas. Med Oncol 29: 1272-1279, 2012.

26. Okada H, Low KL, Kohanbash G, McDonald HA, Hamilton RL and Pollack IF: Expression of glioma-associated antigens in pediatric brain stem and non-brain stem gliomas. J Neurooncol 88: 245-250, 2008

27. Yeung JT, Hamilton RL, Okada H, Jakacki RI and Pollack IF: Increased expression of tumor-associated antigens in pediatric and adult ependymomas: Implication for vaccine therapy. J Neurooncol 111: 103-111, 2013.

28. Skjulsvik AJ, Mørk JN, Torp MO and Torp SH: Ki-67/MIB-1 immunostaining in a cohort of human gliomas. Int J Clin Exp Pathol 7: 8905-8910, 2014.

29. Prayson RA: The utility of MIB-1/Ki-67 immunostaining in the evaluation of central nervous system neoplasms. Adv Anat Pathol 12: 144-148, 2005

30. Johannessen AL and Torp SH: The clinical value of Ki-67/MIB-1 labeling index in human astrocytomas. Pathol Oncol Res 12. 143-147, 2006. 